\title{
ANALYTIC RESULTS FOR THE EIGENVALUES OF CERTAIN TRIDIAGONAL MATRICES*
}

\author{
ALLAN R. WILLMS ${ }^{\dagger}$
}

\begin{abstract}
The eigenvalue problem for a certain tridiagonal matrix with complex coefficients is considered. The eigenvalues and eigenvectors are shown to be expressible in terms of solutions of a certain scalar trigonometric equation. Explicit solutions of this equation are obtained for several special cases, and further analysis of this equation in several other cases provides information about the distribution of eigenvalues.
\end{abstract}

Key words. eigenvalues, eigenvectors, tridiagonal matrices, analytic solutions

AMS subject classification. 15A18

DOI. $10.1137 / 070695411$

1. Introduction. Recently Yueh [9], Kouachi [6], and da Fonseca [4] have studied the eigenvalues of certain tridiagonal matrices, developing a trigonometric equation whose solution yields the eigenvalues. In several special cases this equation has explicit solutions, and exact expressions for the eigenvalues and eigenvectors were obtained. In this paper, we extend their work by more completely describing one further special case where an explicit solution is possible and by analyzing this equation in a number of further cases where information about the distribution of eigenvalues can be obtained.

Consider the tridiagonal matrix

$$
A=\left[\begin{array}{ccccc}
-\alpha+b & c_{1} & & & \\
a_{1} & b & c_{2} & & \\
& a_{2} & \ddots & \ddots & \\
& & \ddots & b & c_{n-1} \\
& & & a_{n-1} & -\beta+b
\end{array}\right]
$$

with the restriction

$$
\sqrt{a_{i} c_{i}}=d \neq 0, \quad 1 \leq i<n .
$$

All variables are, in general, complex. Without loss of generality we may assume that $d$ is the principal square root of $a_{i} c_{i}$ so that its argument is in the range $(-\pi / 2, \pi / 2]$. The matrix $A$ has $n+3$ (complex) degrees of freedom: $a_{i}, 1 \leq i<n, b, d, \alpha$, and $\beta\left(c_{i}\right.$ being determined by $a_{i}$ and $d$ ). However, since the eigenvalues of the matrix $M+b I$ are equal to $\lambda_{i}+b$, where $\lambda_{i}$ are the eigenvalues of the matrix $M$, it follows that the eigenvalues of $A$ will be of the form $\lambda=b+f\left(a_{i}, c_{i}, \alpha, \beta\right)$. It is also not difficult to see that the quantities $a_{i}$ and $c_{i}$ always occur as a product in the characteristic polynomial for $A$, so that the eigenvalues are in fact of the form $\lambda=b+f(d, \alpha, \beta)$. Thus the eigenvalues of $A$ have four complex degrees of freedom. Since $b$ simply appears in an

\footnotetext{
*Received by the editors June 25, 2007; accepted for publication (in revised form) by M. Chu February 5, 2008; published electronically June 13, 2008.

http://www.siam.org/journals/simax/30-2/69541.html

${ }^{\dagger}$ Department of Mathematics and Statistics, University of Guelph, Guelph, ON N1G 2W1, Canada (AWillms@uoguelph.ca).
} 
additive way, we will concentrate on $\mathbb{C}^{3}$, the space of parameters $(d, \alpha, \beta)$. We identify the complex plane with $\mathbb{R}^{2}$, and thus the matrix parameter space is six-dimensional. Most of the special cases yielding explicit solutions for the eigenvalues of $A$ are twodimensional manifolds in this space, although one case is four-dimensional. In addition we will obtain information about the distribution of eigenvalues in various other threeand four-dimensional manifolds in this space.

Yueh [9] considered matrices of the form $A$ but with constant subdiagonal and superdiagonal entries, that is, $a_{i}=a$ and $c_{i}=c, 1 \leq i<n$. He established that the eigenvalues of the $n \times n$ matrix $A$ are of the form $\lambda=b+2 \sqrt{a c} \cos \theta$, and the eigenvectors can also be given in terms of $\theta$, where $\theta$ is a solution of

$$
\text { (3) } \operatorname{ac} \sin ((n+1) \theta)+(\alpha+\beta) \sqrt{a c} \sin (n \theta)+\alpha \beta \sin ((n-1) \theta)=0, \quad \theta \neq k \pi, k \in \mathbb{Z} \text {. }
$$

He solved this equation explicitly in several special cases:

1. $\alpha=\beta=0$. In this case the matrix $A$ is Toeplitz, and the analytic expressions for the eigenvalues and eigenvectors are well known [8, p. 514] to be

$$
\lambda_{m}=b+2 \sqrt{a c} \cos \left(\frac{m \pi}{n+1}\right), \quad v_{j}^{(m)}=\left(\frac{a}{c}\right)^{j-1} \sin \left(\frac{j m \pi}{n+1}\right), \quad 1 \leq m \leq n,
$$

2. $\alpha=0, \beta= \pm \sqrt{a c}$ (or switch $\alpha$ and $\beta$ ),

3. $\alpha=\beta= \pm \sqrt{a c}$,

4. $\alpha=-\beta= \pm \sqrt{a c}$.

The resulting expressions for the eigenvalues and eigenvectors in the last three cases are similar in flavor to the first case [9]. He did not explicitly solve for the special case $\alpha=-c, \beta=-a$. But, by using (3), since $\sin ((n+1) \theta)+\sin ((n-1) \theta)=2 \sin (n \theta) \cos \theta$, for this case we get

$$
\sin (n \theta)=0, \quad \text { or } \quad 2 \sqrt{a c} \cos \theta-(a+c)=0,
$$

which results in eigenvalues

$$
\lambda_{m}=b+2 \sqrt{a c} \cos \left(\frac{m \pi}{n}\right), \quad 1 \leq m<n, \quad \text { and } \quad \lambda_{n}=b+a+c .
$$

For $b=-(a+c)$ this result was known at least as far back as 1954 [7, pp. 365-366].

Kouachi [6] used a different method to study eigenvalues and eigenvectors of the matrix $A$ and generalized to the case where the off-diagonal entries satisfy

$$
a_{i} c_{i}= \begin{cases}d_{1}^{2} & \text { if } i \text { is odd } \\ d_{2}^{2} & \text { if } i \text { is even }\end{cases}
$$

As in Yueh's work, the eigenvalues are given in terms of $\cos \theta$ and a nonlinear equation involving $\theta$ is specified. In addition to the special cases considered by Yueh, Kouachi also found explicit expressions for the eigenvalues and eigenvectors in the case $\alpha \beta=d_{2}^{2}, n$ even. This amounts to a generalization of Ledermann and Reuter's [7] result, although only for even $n$, and, unfortunately, there are a number of substantial typographical errors in Kouachi's paper particularly in the exposition of this case.

The eigenvalues of certain perturbed tridiagonal $k$-Toeplitz matrices were recently studied by da Fonseca [4]. In particular, da Fonesca gives a trigonometric relation satisfied by the eigenvalues of a 2-Toeplitz matrix with perturbed entries in the top left and bottom right corners. This result is more general than those of Yueh and 
Kouachi, and those presented here, since the matrix $A$ and those studied by Yueh and Kouachi are special types of 2-Toeplitz matrices. However, da Fonseca gives an explicit formula for the eigenvalues in just one special case of the perturbation parameters $(\alpha$ and $\beta)$ but no others. (Incidentally, there is a typographical error in his paper for this formula also: $b_{1}^{2}+b_{2}^{2}$ on page 65 should be $b_{1}+b_{2}$.)

We also note another recent result by da Fonseca [5] which relates the eigenvalues of the matrix

$$
C=[\mu \min \{i, j\}-\nu]_{i, j=1, \ldots, n}, \quad \mu>0, \quad \mu \neq \nu,
$$

to those of a matrix of the form $A$ (1) with $a_{i}=c_{i}=-1, i=1, \ldots, n-1, b=2$, $\alpha=1-\mu /(\mu-\nu)$, and $\beta=1$. However, he obtains explicit results only for a couple of special values for $\mu$ and $\nu$, which turn out to be two of the special cases that we consider here.

In this paper we provide a general expression for the eigenvalues and eigenvectors of the matrix $A$, which generalizes Yueh's result and is contained within Kouachi's and da Fonseca's. We enumerate all of the special cases where this general expression yields explicit results for the eigenvalues and eigenvectors; particularly, we describe the case $\alpha \beta=d^{2}$ fully, extending the work of Yueh and Kouachi. In addition, we provide analysis in several other special cases not considered by any of the above three authors where explicit solutions are not possible but information on the distribution of eigenvalues can be obtained.

2. General results. Similar to Yueh [9], we utilize the methods of symbolic calculus of semi-infinite sequences [3]. The pertinent facts are as follows. The convolution of two sequences $x=\left\{x_{j}\right\}_{j=0}^{\infty}$ and $y=\left\{y_{j}\right\}_{j=0}^{\infty}$ is the sequence $z=\left\{z_{n}\right\}_{n=0}^{\infty}$ whose $n$th component is

$$
z_{n}=\sum_{j=0}^{n} x_{j} y_{n-j} .
$$

Convolution is a symmetric operation $x y=y x$, distributes over addition $x(y+z)=$ $x y+x z$, and is associative with respect to scalar multiplication $x(c y)=c(x y)$ for any scalar $c$. We define $I=\{1,0, \ldots\}$ and $S=\{0,1,0, \ldots\}$. Thus

$$
I x=x \quad \text { and } \quad S x=\left\{0, x_{0}, x_{1}, \ldots\right\}
$$

and, in particular,

$$
S\left\{x_{j+1}\right\}_{j=0}^{\infty}=\left\{0, x_{1}, x_{2}, \ldots\right\}=x-x_{0} I
$$

and

$$
S^{2}\left\{x_{j+2}\right\}_{j=0}^{\infty}=\left\{0,0, x_{2}, x_{3}, \ldots\right\}=x-x_{0} I-x_{1} S .
$$

Each sequence $x$ with $x_{0} \neq 0$ has a unique inverse $y$ such that $x y=I$, where $y_{0}=1 / x_{0}$ and $y_{n}, n>0$, is defined recursively by $y_{n}=-\left(\sum_{j=1}^{n} x_{j} y_{n-j}\right) / x_{0}$. In particular, the inverse of $(S-\gamma I)$, for scalar $\gamma$, is

$$
(S-\gamma I)^{-1}=\left\{\frac{-1}{\gamma^{j+1}}\right\}_{j=0}^{\infty} .
$$


Consider now the eigenproblem for the matrix $A, A v=\lambda v$, which can be written as follows:

$$
\begin{aligned}
(b-\lambda) v_{1}+c_{1} v_{2} & =\alpha v_{1}, \\
a_{1} v_{1}+(b-\lambda) v_{2}+c_{2} v_{3} & =0,
\end{aligned}
$$

$$
\begin{aligned}
a_{n-2} v_{n-2}+(b-\lambda) v_{n-1}+c_{n-1} v_{n} & =0 \\
a_{n-1} v_{n-1}+(b-\lambda) v_{n} & =\beta v_{n}
\end{aligned}
$$

Since $d^{2}=a_{j} c_{j}$, for all $j=1, \ldots, n-1$, it follows that $d / c_{j}=a_{j} / d$. Define $q_{1}=1$ and $q_{j}, 1<j \leq n$, by the recursion

$$
q_{j}=\frac{d}{c_{j-1}} q_{j-1}=\frac{a_{j-1}}{d} q_{j-1}, \quad 1<j \leq n .
$$

Formally, the $q_{j}$ are given by

$$
q_{j}=\left(\frac{1 a_{1} a_{2} \cdots a_{j-1}}{1 c_{1} c_{2} \cdots c_{j-1}}\right)^{1 / 2} ;
$$

however, the specific branch of the square root function that needs to be used for each subsequent $j$ is not always the principal branch but rather is determined by the requirement that $q_{j}=d q_{j-1} / c_{j-1}$. Note that, by $(2), a_{j}$ and $c_{j}$, and hence $q_{j}$, are all nonzero so that the vector $u$ given by

$$
v_{j}=q_{j} u_{j}, \quad 1 \leq j \leq n,
$$

is well defined. Substitute (8) into system (5), dividing the $j$ th equation by $q_{j}$. By using (6) this process yields the system

$$
\begin{aligned}
(b-\lambda) u_{1}+d u_{2} & =\alpha u_{1}, \\
d u_{1}+(b-\lambda) u_{2}+d u_{3} & =0,
\end{aligned}
$$

$$
\begin{aligned}
d u_{n-2}+(b-\lambda) u_{n-1}+d u_{n} & =0, \\
d u_{n-1}+(b-\lambda) u_{n} & =\beta u_{n} .
\end{aligned}
$$

We now extend the vector $u$ to a semi-infinite sequence $\left\{u_{j}\right\}_{j=0}^{\infty}$ and impose $u_{0}=0$ and $u_{n+1}=0$. System (9) can then be written as

$$
d\left\{u_{j+2}\right\}_{j=0}^{\infty}+(b-\lambda)\left\{u_{j+1}\right\}_{j=0}^{\infty}+d\left\{u_{j}\right\}_{j=0}^{\infty}=\left\{f_{j+1}\right\}_{j=0}^{\infty},
$$

where $f$ is the sequence defined by

$$
f_{j}= \begin{cases}\alpha u_{1} & \text { if } j=1, \\ \beta u_{n} & \text { if } j=n, \\ 0 & \text { otherwise. }\end{cases}
$$

Taking the convolution of (10) with $S^{2}$ gives

$$
d\left(u-u_{0} I-u_{1} S\right)+(b-\lambda) S\left(u-u_{0} I\right)+d S^{2} u=S\left(f-f_{0} I\right),
$$

Copyright (C) by SIAM. Unauthorized reproduction of this article is prohibited. 
and by using the facts that $u_{0}=0$ and $f_{0}=0$ we have

$$
\left(d S^{2}+(b-\lambda) S+d I\right) u=\left(f+d u_{1} I\right) S .
$$

Let

$$
\gamma_{ \pm}=\frac{1}{2 d}[-(b-\lambda) \pm \sqrt{\omega}]
$$

where $\omega=(b-\lambda)^{2}-4 d^{2}$, be the two roots of $d x^{2}+(b-\lambda) x+d=0$. Note that $\gamma_{+} \gamma_{-}=1$, and thus we may write $\gamma_{ \pm}=e^{ \pm i \theta}$, where $\theta \in \mathbb{C}$ by taking $\theta=\arg \gamma_{+}-i \ln \left|\gamma_{+}\right|$. Since $-(b-\lambda) / d=\gamma_{+}+\gamma_{-}=e^{i \theta}+e^{-i \theta}=2 \cos \theta$, the eigenvalues are given by

$$
\lambda=b+2 d \cos \theta .
$$

Further, since $\cos (x+i y)=\cosh (y) \cos (a)-i \sinh (b) \sin (a)$, it follows that the eigenvalue is real either if $\theta$ is real or if $\operatorname{Re}(\theta)=k \pi, k \in \mathbb{Z}$. With this notation, (12) becomes

$$
d\left(S-\gamma_{+} I\right)\left(S-\gamma_{-} I\right) u=\left(f+d u_{1} I\right) S
$$

and from (4) we obtain

$$
\begin{aligned}
& u=\left\{\frac{-1}{\gamma_{+}^{j+1}}\right\}_{j=0}^{\infty}\left\{\frac{-1}{\gamma_{-}^{j+1}}\right\}_{j=0}^{\infty}\left(\frac{1}{d} f+u_{1} I\right) S \\
& =\left\{\sum_{j=0}^{m} \frac{1}{\gamma_{+}^{j+1} \gamma_{-}^{m-j+1}}\right\}_{m=0}^{\infty}\left(\frac{1}{d} f+u_{1} I\right) S \\
& =\left\{\sum_{j=0}^{m} \gamma_{-}^{j} \gamma_{+}^{m-j}\right\}_{m=0}^{\infty}\left(\frac{1}{d} f+u_{1} I\right) S \\
& = \begin{cases}\left\{\frac{\gamma_{+}^{m+1}-\gamma_{-}^{m+1}}{\gamma_{+}-\gamma_{-}}\right\}_{m=0}^{\infty}\left(\frac{1}{d} f+u_{1} I\right) S & \text { if } \gamma_{+} \neq \gamma_{-}, \\
\left\{(m+1) \gamma_{+}^{m}\right\}_{m=0}^{\infty}\left(\frac{1}{d} f+u_{1} I\right) S & \text { if } \gamma_{+}=\gamma_{-},\end{cases} \\
& = \begin{cases}\left\{\frac{\sin ((m+1) \theta)}{\sin \theta}\right\}_{m=0}^{\infty}\left(\frac{1}{d} f+u_{1} I\right) S & \text { if } \gamma_{+} \neq \gamma_{-}, \\
\left\{(m+1) e^{i m \theta}\right\}_{m=0}^{\infty}\left(\frac{1}{d} f+u_{1} I\right) S & \text { if } \gamma_{+}=\gamma_{-} .\end{cases}
\end{aligned}
$$

If we define the function $g: \mathbb{Z} \times \mathbb{C} \rightarrow \mathbb{C}$ as

$$
g(n, \theta)= \begin{cases}\frac{\sin (n \theta)}{\sin \theta} & \text { if } \theta \neq k \pi, k \in \mathbb{Z}, \\ n & \text { if } \theta=2 k \pi, k \in \mathbb{Z}, \\ (-1)^{n-1} n & \text { if } \theta=(2 k+1) \pi, k \in \mathbb{Z},\end{cases}
$$

Copyright $@$ by SIAM. Unauthorized reproduction of this article is prohibited. 
then $g$ is continuous in $\theta$, and, since $\gamma_{+}=\gamma_{-}$is equivalent to $\theta=k \pi, k \in \mathbb{Z}$, both of the cases in (15) collapse to

$$
u=\{g(m+1, \theta)\}_{m=0}^{\infty}\left(\frac{1}{d} f+u_{1} I\right) S
$$

Computing the convolutions in this last expression and noting from (11) that the only nonzero entries of the sequence $f$ are $f_{1}=\alpha u_{1}$ and $f_{n}=\beta u_{n}$ yields $u_{0}=0$ and

$$
u_{j}=u_{1}\left[g(j, \theta)+\frac{\alpha}{d} g(j-1, \theta)\right]+H(j-n-1) \frac{\beta}{d} u_{n} g(j-n, \theta), \quad j \geq 1,
$$

where $H(x)$ is the unit step function: $H(x)=1$ if $x \geq 0$, and $H(x)=0$ if $x<0$. By using (18) with $j=n+1$, the condition $u_{n+1}=0$ becomes

$$
\left[g(n+1, \theta)+\frac{\alpha}{d} g(n, \theta)\right] u_{1}+\frac{\beta}{d} u_{n}=0,
$$

where we have used the fact that $g(1, \theta)=1$. Finally, by using (18) at $j=n$ and noting that $u_{1}$ cannot be zero (otherwise, the vector $v$ must be zero) we obtain the necessary and sufficient condition for $\lambda=b+2 d \cos \theta$ to be an eigenvalue of $A$, namely,

$$
g(n+1, \theta)+\frac{\alpha+\beta}{d} g(n, \theta)+\frac{\alpha \beta}{d^{2}} g(n-1, \theta)=0 .
$$

Equations (20) and (14) correspond to those derived by Yueh; however, he multiplied (20) by $d^{2} \sin \theta$ to clear the denominator and dealt with the case $\theta=k \pi$ separately.

We now show that there are exactly $n$ solutions (counting multiplicity) of (20) in the region

$$
R=\{\theta=(x+i y) \mid 0 \leq x \leq \pi, x, y \in \mathbb{R}\},
$$

where roots on the boundary of $R$ are counted with half weight. The continuous function $g$ is $2 \pi$-periodic in $\theta$ and is an even function of $\theta$. Consequently once the roots of (20) in $R$ are found, all roots of (20) can be determined. Setting $\xi=e^{i \theta}$ and multiplying (20) by the nonzero quantity $d^{2} \xi^{n+1} / \xi$ gives

$$
\frac{d^{2}\left(\xi^{2 n+2}-1\right)+d(\alpha+\beta) \xi\left(\xi^{2 n}-1\right)+\alpha \beta \xi^{2}\left(\xi^{2 n-2}-1\right)}{\xi^{2}-1}=0 .
$$

Clearly $\xi= \pm 1$ are roots of the numerator; hence, $\xi^{2}-1$ divides the numerator, and we are left with a $2 n$th order polynomial in $\xi$ which necessarily has $2 n$ roots, some possibly repeated. Since $e^{i \theta}$ is $2 \pi$-periodic, it follows that there are $2 n$ roots of $(20)$ in the region $R \cup \hat{R}$, where $\hat{R}=\{\theta \mid-\pi<\operatorname{Re}(\theta)<0\}$. By the even property of $g$, every root in $\operatorname{int}(R)$ (the interior of $R$ ) has a corresponding root in $\hat{R}$. Since $R$ and $\hat{R}$ are disjoint, it follows that twice the number or roots in $\operatorname{int}(R)$ plus the number of roots on the boundary of $R$ is $2 n$. Further, roots on the left boundary of $R$ of the form $\theta=i y, y>0$, have corresponding roots $\theta=-i y$ also on the left boundary, and similarly roots on the right boundary of $R$ of the form $\theta=\pi+i y, y>0$, have corresponding roots $\theta=-\pi-i y+2 \pi=\pi-i y$, also on the right boundary. Since the cosine is also even and $2 \pi$-periodic, each of these corresponding pairs of roots of (20) yield identical eigenvalues of $A$ through (14). We may thus focus entirely on finding 
roots of (20) in the region $R$ and may also exclude the portions of the boundary of $R$ with $\operatorname{Im}(\theta)<0$. Each such distinct root $\theta$ corresponds to a distinct eigenvalue $\lambda$.

From (18) and (8), the components of the eigenvectors are given by

$$
v_{j}=v_{1} q_{j}\left[g(j, \theta)+\frac{\alpha}{d} g(j-1, \theta)\right], \quad 1<j \leq n .
$$

But, since $q_{1}=1, g(0, \theta)=0$, and $g(1, \theta)=1$, we may express the eigenvectors as

$$
v_{j}=\left\{\begin{array}{ll}
q_{j}\left[\sin (j \theta)+\frac{\alpha}{d} \sin ((j-1) \theta)\right] & \text { if } \theta \notin\{0, \pi\}, \\
q_{j}\left[j+\frac{\alpha}{d}(j-1)\right] & \text { if } \theta=0, \\
q_{j}(-1)^{j-1}\left[j-\frac{\alpha}{d}(j-1)\right] & \text { if } \theta=\pi,
\end{array} \quad 1 \leq j \leq n .\right.
$$

3. Special cases. In this section we examine various relationships between the matrix parameters $d, \alpha$, and $\beta$ which, when enforced, allow (20) to be solved explicitly or to be simplified to the form $F(\theta)=p(d, \alpha, \beta)$. These simplifications are the result of standard trigonometric identities. We list here several simplifications that we use for our function $g$ :

$$
\begin{aligned}
g(j+1, \theta)+g(j-1, \theta) & =2 g(j, \theta) \cos \theta, \\
g(j+1, \theta)-g(j-1, \theta) & =2 \cos (j \theta), \\
g(j, \theta)+g(j-1, \theta) & =g(2 j-1, \theta / 2), \\
g(j, \theta)-g(j-1, \theta) & = \begin{cases}\cos ((2 j-1) \theta / 2) / \cos (\theta / 2) & \text { if } 0<\theta<\pi, \\
1 & \text { if } \theta=0, \\
(-1)^{j-1}(2 j-1) & \text { if } \theta=\pi .\end{cases}
\end{aligned}
$$

Note that all of the above are identities for $g$ and are valid for all $\theta \in \mathbb{C}$, including $\theta=k \pi, k \in \mathbb{Z}$.

\subsection{Explicit solutions.}

3.1.1. $\alpha=\boldsymbol{\beta}=\mathbf{0}$. If $\alpha=\beta=0$, then the matrix $A$, although not Toeplitz, has the same eigenvalues as the corresponding Toeplitz matrix $\left(a_{i}=a\right.$ and $c_{i}=c$ for all $i)$, since (20) collapses to $g(n+1, \theta)=0$, whose solutions are $\theta_{m}=\frac{m \pi}{n+1}, 1 \leq m \leq n$, giving eigenvalues

$$
\lambda_{m}=b+2 d \cos \left(\frac{m \pi}{n+1}\right), \quad 1 \leq m \leq n,
$$

with corresponding eigenvectors

$$
v_{j}^{(m)}=q_{j} \sin \left(\frac{j m \pi}{n+1}\right), \quad 1 \leq j \leq n, 1 \leq m \leq n .
$$

3.1.2. $\boldsymbol{\alpha}=\mathbf{0}, \boldsymbol{\beta}=\boldsymbol{d}$. If $\alpha=0, \beta=d$, then by using (27), (20) becomes

$$
g(2 n+1, \theta / 2)=0
$$

whose solutions are $\theta_{m}=\frac{2 m \pi}{2 n+1}$, giving eigenvalues

$$
\lambda_{m}=b+2 d \cos \left(\frac{2 m \pi}{2 n+1}\right), \quad 1 \leq m \leq n,
$$

and corresponding eigenvectors

$$
v_{j}^{(m)}=q_{j} \sin \left(\frac{2 m j \pi}{2 n+1}\right), \quad 1 \leq j \leq n, 1 \leq m \leq n .
$$

Copyright $@$ ( ) by SIAM. Unauthorized reproduction of this article is prohibited. 
3.1.3. $\boldsymbol{\alpha}=\boldsymbol{d}, \boldsymbol{\beta}=\mathbf{0}$. If $\alpha=d, \beta=0$, the eigenvalues are the same as the above case (31), and the eigenvectors are

$$
v_{j}^{(m)}=q_{j} \cos \left(\frac{(2 j-1) 2 m \pi}{2(2 n+1)}\right), \quad 1 \leq j \leq n, 1 \leq m \leq n .
$$

3.1.4. $\boldsymbol{\alpha}=\mathbf{0}, \boldsymbol{\beta}=-\boldsymbol{d}$. If $\alpha=0, \beta=-d$, we may use (28) to reduce (20) to

$$
\cos ((2 n+1) \theta / 2)=0,
$$

whose solutions are $\theta_{m}=(2 m-1) \pi /(2 n+1), 1 \leq m \leq n$, giving eigenvalues

$$
\lambda_{m}=b+2 d \cos \left(\frac{(2 m-1) \pi}{2 n+1}\right), \quad 1 \leq m \leq n,
$$

and corresponding eigenvectors

$$
v_{j}^{(m)}=q_{j} \sin \left(\frac{j(2 m-1) \pi}{2 n+1}\right), \quad 1 \leq j \leq n, 1 \leq m \leq n .
$$

3.1.5. $\alpha=-\boldsymbol{d}, \boldsymbol{\beta}=\mathbf{0}$. If $\alpha=-d, \beta=0$, the eigenvalues are the same as the previous case (34), and the corresponding eigenvectors are

$$
v_{j}^{(m)}=q_{j} \cos \left(\frac{(2 j-1)(2 m-1) \pi}{2(2 n+1)}\right), \quad 1 \leq j \leq n, 1 \leq m \leq n .
$$

3.1.6. $\boldsymbol{\alpha}=\boldsymbol{d}, \boldsymbol{\beta}=-\boldsymbol{d}$. If $\alpha=-\beta=d$, (20) simplifies by using (26) to become $\cos (n \theta)=0$. Hence $\theta_{m}=\frac{(2 m-1) \pi}{2 n}, 1 \leq m \leq n$, and the eigenvalues are

$$
\lambda_{m}=b+2 d \cos \left(\frac{(2 m-1) \pi}{2 n}\right), \quad 1 \leq m \leq n,
$$

with corresponding eigenvectors

$$
v_{j}^{(m)}=q_{j} \sin \left(\frac{(2 j-1)(2 m-1) \pi}{4 n}\right), \quad 1 \leq j \leq n, 1 \leq m \leq n .
$$

3.1.7. $\alpha=-\boldsymbol{d}, \boldsymbol{\beta}=\boldsymbol{d}$. If $\alpha=-\beta=-d$, the eigenvalues are the same as the previous case (37), and the corresponding eigenvectors are

$$
v_{j}^{(m)}=q_{j} \cos \left(\frac{(2 j-1)(2 m-1) \pi}{4 n}\right), \quad 1 \leq j \leq n, 1 \leq m \leq n .
$$

3.1.8. $\boldsymbol{\alpha} \boldsymbol{\beta}=\boldsymbol{d}^{2}$. Now consider the case $\alpha \beta=d^{2}$. Whereas the previous special cases were two-dimensional real manifolds in $(d, \alpha, \beta)$-space, this case is a fourdimensional manifold in $\mathbb{R}^{6}$. By using (25), (20) becomes

$$
g(n, \theta)\left(2 \cos \theta+\frac{\alpha+\beta}{d}\right)=0 .
$$

From this we immediately obtain

$$
\theta_{m}=\frac{m \pi}{n}, \quad 1 \leq m<n, \quad \text { or } \quad 2 d \cos \theta_{n}=-(\alpha+\beta),
$$


giving eigenvalues

$$
\lambda_{m}=b+2 d \cos \left(\frac{m \pi}{n}\right), \quad 1 \leq m<n, \quad \text { and } \quad \lambda_{n}=b-(\alpha+\beta) .
$$

The more specialized cases with $\alpha=\beta=d$ or $\alpha=\beta=-d$ were the only ones with $\alpha \beta=d^{2}$ that were considered by Yueh. In the case $\alpha=\beta=d$, by using (24) and (27), the eigenvectors are

$$
v_{j}^{(m)}=q_{j} \sin \left(\frac{(2 j-1) m \pi}{2 n}\right), \quad 1 \leq j \leq n, 1 \leq m<n,
$$

and, for the $n$th eigenpair, $\theta_{n}=\pi, \lambda_{n}=b-2 d$, and

$$
v_{j}^{(n)}=q_{j}(-1)^{j-1}, \quad 1 \leq j \leq n,
$$

which we note is the same form as (41) with $m=n$. The case $\alpha=\beta=-d$ gives eigenvectors

$$
v_{j}^{(m)}=q_{j} \cos \left(\frac{(2 j-1) m \pi}{2 n}\right), \quad 1 \leq j \leq n, 1 \leq m<n,
$$

and $\theta_{n}=0, \lambda_{n}=b+2 d$, and, by using (28),

$$
v_{j}^{(n)}=q_{j}, \quad 1 \leq j \leq n .
$$

Outside these more specialized cases, it is impossible for $\theta_{n}$ to be zero or $\pi$, and so, from (24), we may write the eigenvectors as

$$
v_{j}^{(m)}=q_{j}\left[\sin \left(\frac{j m \pi}{n}\right)+\frac{\alpha}{d} \sin \left(\frac{(j-1) m \pi}{n}\right)\right], \quad 1 \leq j \leq n, 1 \leq m<n,
$$

and

$$
v_{j}^{(n)}=q_{j}\left[\sin \left(j \theta_{n}\right)+\frac{\alpha}{d} \sin \left((j-1) \theta_{n}\right)\right], \quad 1 \leq j \leq n,
$$

where $\theta_{n}=\arccos (-(\alpha+\beta) / 2 d)$.

3.2. Eigenvalue distribution results. Here we examine various three- and four-dimensional real manifolds in $(d, \alpha, \beta)$-space, where information on the distribution of the eigenvalues can be inferred. For this analysis it is convenient to partition the interval $[0, \pi]$ into subintervals in one of several ways. In the first way, $[0, \pi]$ is partitioned into $n+1$ subintervals, the first and last of which have width $1 /(2 n)$ while the remaining have width $1 / n$ :

$$
\begin{aligned}
I_{0} & =\left[0, \frac{\pi}{2 n}\right) \\
I_{k} & =\left[\frac{(2 k-1) \pi}{2 n}, \frac{(2 k+1) \pi}{2 n}\right), \quad 1 \leq k<n-1, \\
I_{n} & =\left[\frac{(2 n-1) \pi}{2 n}, \pi\right] .
\end{aligned}
$$

Copyright $@$ by SIAM. Unauthorized reproduction of this article is prohibited. 


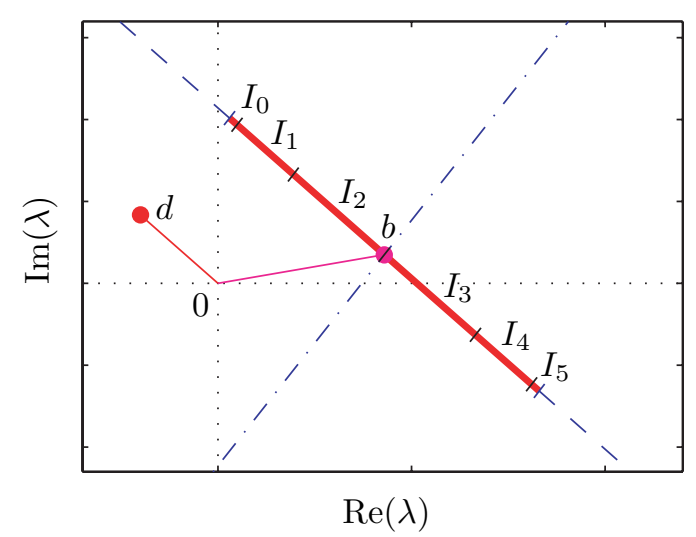

FIG. 1. The location of eigenvalues $\lambda=b+2 d \cos (\theta)$ in the complex plane for various $\theta$. If $\theta \in[0, \pi]$, then $\lambda$ lies on the thick solid line segment. For illustration, this segment is divided into the images of the intervals $I_{k}, 0 \leq k \leq n, n=5$. Other partitionings of $[0, \pi]$ would divide the line segment differently. The dashed line extending beyond this line segment shows the location of $\lambda$ when $\theta=i y$ or $\theta=\pi+i y, y \in \mathbb{R}$. The dotted-dashed line orthogonal to the thick line segment is the image of the line $\theta=\pi / 2 \pm i y, y \in \mathbb{R}$.

A second useful partitioning of $[0, \pi]$ is one where the subintervals have width $2 /(2 n+$ 1) except for the first, which has half that width:

$$
\begin{aligned}
J_{0} & =\left[0, \frac{\pi}{2 n+1}\right) \\
J_{k} & =\left[\frac{(2 k-1) \pi}{2 n+1}, \frac{(2 k+1) \pi}{2 n+1}\right), \quad 1 \leq k<n-1, \\
J_{n} & =\left[\frac{(2 n-1) \pi}{2 n+1}, \pi\right] .
\end{aligned}
$$

Finally, consider partitioning $[0, \pi]$ into $n$ equal length intervals:

$$
\begin{aligned}
K_{k} & =\left[\frac{(k-1) \pi}{n}, \frac{k \pi}{n}\right), \quad 1 \leq k<n, \\
K_{n} & =\left[\frac{(n-1) \pi}{n}, \pi\right] .
\end{aligned}
$$

Typically, the following results make statements such as "Under the stated assumptions, there is one real solution of (20) lying in each of the intervals $I_{0}$ to $I_{n-1}$." The eigenvalues of $A$ are given by $\lambda=b+2 d \cos (\theta)$. Real values of $\theta$ on the interval $[0, \pi]$ thus correspond to eigenvalues on a line segment of length $4|d|$ parallel to the ray from the origin to $d$ and centered at $b$, as depicted in Figure 1. The various subintervals defined above correspond to portions of this line segment. Sometimes complex solutions for $\theta$ of the form $\theta=i y, \theta=\pi+i y$, or $\theta=\pi / 2 \pm i y, y \in \mathbb{R}$, are also shown to exist. In the first two cases, the corresponding eigenvalues are $\lambda=b \pm 2 d \cosh (y)$, respectively, which lie on the same line as the intervals shown in Figure 1 but further away from $b$. In the case $\theta=\pi / 2 \pm i y$, the corresponding eigenvalues are $\lambda=b \pm i 2 d \sinh (y)$, which lie an equal distance from $b$ on a line through $b$ orthogonal to the ray through $d$. Of course, if $b$ and $d$ are real, then each of these distinct real solutions for $\theta$ and the complex solutions with $\operatorname{Re}(\theta) \in\{0, \pi\}$ yield distinct real eigenvalues for $A$. 


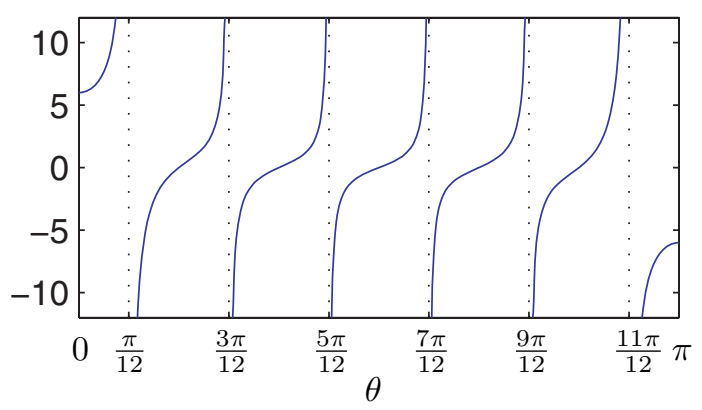

FIG. 2. The function $\theta \mapsto \tan (n \theta) / \sin (\theta), \theta \in[0, \pi]$, for the case $n=6$. The intervals $I_{k}$, $0 \leq k \leq n$, are demarcated by the dotted vertical lines.

For some of this analysis it is convenient to express the necessary and sufficient condition for $\theta,(20)$, in an equivalent form. By expanding $\sin ((n \pm 1) \theta)$ and simplifying, (20) becomes

$$
\frac{\sin (n \theta)}{\sin (\theta)}\left[\left(1+\frac{\alpha \beta}{d^{2}}\right) \cos (\theta)+\frac{\alpha+\beta}{d}\right]+\cos (n \theta)\left(1-\frac{\alpha \beta}{d^{2}}\right)=0 .
$$

3.2.1. $\boldsymbol{\alpha} \boldsymbol{\beta}=-\boldsymbol{d}^{2}$. Consider the four-dimensional manifold $\alpha \beta=-d^{2}$, but exclude the cases $\alpha=-\beta= \pm d$ as these have already been considered. In this case, (50) simplifies to

$$
\frac{\sin (n \theta)}{\sin (\theta)}\left[\frac{\alpha+\beta}{d}\right]+2 \cos (n \theta)=0
$$

Since we have excluded the possibility that $\alpha+\beta=0$, for (51) to hold $\cos (n \theta)$ cannot be zero, and hence we may write this equation as

$$
\frac{\tan (n \theta)}{\sin (\theta)}=\frac{-2 d}{\alpha+\beta}
$$

Let $F(\theta)=\tan (n \theta) / \sin (\theta)$ and $p(d, \alpha, \beta)=-2 d /(\alpha+\beta)$, and assume that the value of $p$ is real. (We are now restricted to a three-dimensional manifold.) The function $F$ on $[0, \pi]$ is an odd function with respect to $\pi / 2$. It monotonically increases from $-\infty$ to $+\infty$ on each interval $I_{k}, 1 \leq k<n$. On the interval $I_{0}$ it monotonically increases from $n$ to $+\infty$, and on the interval $I_{n}$ it monotonically increases from $-\infty$ to $-n$; see Figure 2. Consequently, if $|p| \geq n$, there are exactly $n$ distinct real solutions of (52) for $\theta$ in $[0, \pi]$, one in each of the intervals $I_{1}, \ldots, I_{n-1}$ and the last one in either $I_{0}$ (if $p>0$ ) or in $I_{n}$ (if $p<0$ ). These $n$ distinct values for $\theta$ correspond to $n$ distinct real eigenvalues $\lambda$. If $|p|<n$, then there are only $n-1$ real solutions of (52), one in each of the intervals $I_{1}, \ldots, I_{n-1}$, corresponding to $n-1$ real eigenvalues for $A$. In the event that all variables in $A$ are real, the last eigenvalue must also be real, since they must occur in complex conjugate pairs. (It turns out that, even if just $b, d$, and $p$ are real, the last eigenvalue is also real.) But for $\theta=x+i y, x, y \in \mathbb{R}$,

$$
\cos (\theta)=\cos (x+i y)=\cosh (y) \cos (x)-i \sinh (y) \sin (x),
$$

thus $\lambda=b+2 d \cos (\theta)$ will be real if $b$ and $d$ are real, and $\theta$ is real or $\operatorname{Re}(\theta)=k \pi$. We are thus led to look for the $n$th solution of (52) on the boundary of $R$ by taking 


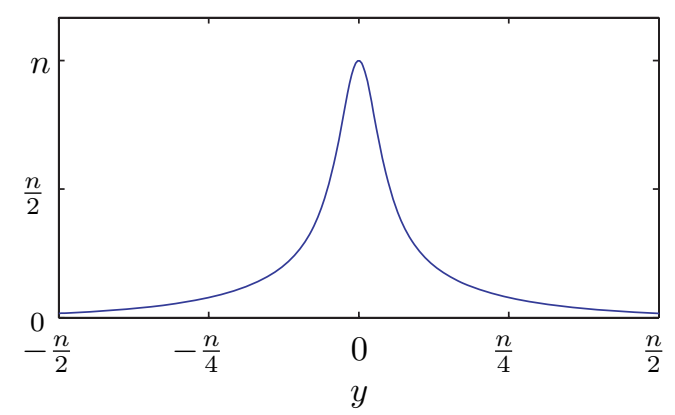

FIG. 3. The function $y \mapsto \tanh (n y) / \sinh (y)$.

$\theta=i y$ or $\theta=\pi+i y, y>0$. (Recall that on the boundary of $R$ we need only consider $y \geq 0$.) If $0<p<n$, substituting $\theta=i y, y \in \mathbb{R}$, into (52) gives

$$
\frac{\tanh (n y)}{\sinh (y)}=p
$$

A plot of this function is shown in Figure 3. Since it monotonically decreases from $n$ to zero as $y$ increases from zero, there is exactly one solution $y^{*}$ of (53), with $y^{*}>0$. If $-n<p<0$, substituting $\theta=\pi+i y, y \in \mathbb{R}$, into (52) gives

$$
\frac{\tanh (n y)}{\sinh (y)}=-p
$$

again yielding exactly one more root $y^{*}$, with $y^{*}>0$. If $|p|$ is large $(|\alpha+\beta|$ is small), then the solutions for $\theta$ asymptotically approach $\theta_{m}=(2 m-1) \pi /(2 n), 1 \leq m \leq n$, as expected since these are the solutions for $\alpha=-\beta= \pm d$. But in any event the real solutions of (52) are approximately spaced by a distance of $\pi / n$ as is readily apparent in Figure 2.

3.2.2. $\boldsymbol{\alpha} \boldsymbol{\beta}=\mathbf{0}$. If the product $\alpha \beta$ is zero (but $\alpha$ and $\beta$ are not both zero and one is not $\pm d$, as these cases have been previously considered), then (20) becomes

$$
g(n+1, \theta)+\frac{\alpha+\beta}{d} g(n, \theta)=0 .
$$

This can be written as

$$
\frac{\sin ((n+1 / 2) \theta+\theta / 2)}{\sin (2 \theta / 2)}+\left(\frac{\alpha+\beta}{d}\right) \frac{\sin ((n+1 / 2) \theta-\theta / 2)}{\sin (2 \theta / 2)}=0,
$$

which upon expanding and simplifying yields

$$
\left(1+\frac{\alpha+\beta}{d}\right)\left[\frac{\sin ((2 n+1) \theta / 2)}{\sin (\theta / 2)}\right]+\left(1-\frac{\alpha+\beta}{d}\right)\left[\frac{\cos ((2 n+1) \theta / 2)}{\cos (\theta / 2)}\right]=0 .
$$

Now, since we are assuming that $\alpha+\beta \neq \pm d$, both factors in parentheses in the above expression are nonzero. This means that if one of the factors in square brackets is zero, both must be zero for the equation to hold. However, this is impossible since the first factor in square brackets is $g(2 n+1, \theta / 2)$, which is zero only when $(2 n+1) \theta / 2=k \pi$, 


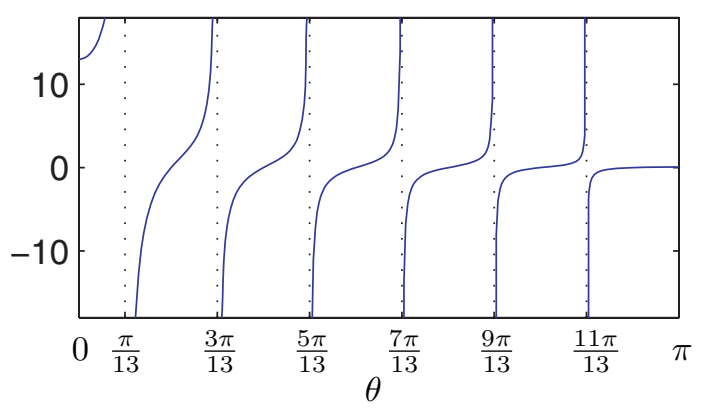

FIG. 4. The function $\theta \mapsto \tan ((2 n+1) \theta / 2) / \tan (\theta / 2), \theta \in[0, \pi]$, for the case $n=6$. The intervals $J_{k}, 0 \leq k \leq n$, are demarcated by the dotted vertical lines.

$1 \leq k \leq n$, which implies that $\cos ((2 n+1) \theta / 2)=\cos (k \pi) \neq 0$. We may thus validly rearrange the above expression to obtain

$$
\frac{\tan ((2 n+1) \theta / 2)}{\tan (\theta / 2)}=\frac{\alpha+\beta-d}{\alpha+\beta+d} .
$$

Let $F(\theta)$ and $p(d, \alpha, \beta)$ be the left and right sides of the above equation, respectively, and assume that the value of $p$ is real. (Again, we are now restricted to a threedimensional manifold.) The function $F$ on $[0, \pi]$ is plotted in Figure 4 for the case $n=6 . F$ is monotonically increasing from $-\infty$ to $+\infty$ on the intervals $J_{k}, 1 \leq k<n$, monotonically increasing from $2 n+1$ to $+\infty$ on $J_{0}$, and monotonically increasing from $-\infty$ to $1 /(2 n+1)$ on $J_{n}$. Thus if $p$ is real and at least as big as $2 n+1$, there are exactly $n$ real solutions, one in each of the intervals $J_{k}, 0 \leq k \leq n-1$. If $p$ is real and equal to or smaller than $1 /(2 n+1)$, there is one real solution in each of the intervals $J_{k}, 1 \leq k \leq n$. All of these real roots are approximately separated by a distance of $2 \pi /(2 n+1)$. Substituting $\theta=i y, y \in \mathbb{R}$, into (56) gives

$$
\frac{\tanh ((2 n+1) y / 2)}{\tanh (y / 2)}=p
$$

while substituting $\theta=\pi+i y, y \in \mathbb{R}$, into (56) gives

$$
\frac{\operatorname{coth}((2 n+1) y / 2)}{\operatorname{coth}(y / 2)}=p .
$$

The functions on the left sides of the above expressions are shown in Figure 5 . The first decreases monotonically from $2 n+1$ to 1 , while the second increases from $1 /(2 n+1)$ to 1 as $y$ increases from zero. Thus if $1 /(2 n+1)<p<1$, there is one solution of (56) of the form $\theta=\pi+i y^{*}, y^{*}>0$, and if $1<p<(2 n+1)$, there is one solution of the form $\theta=i y^{*}, y^{*}>0$. (Note that since $d \neq 0$ it is impossible for $p=1$.)

3.2.3. $\boldsymbol{\alpha}+\boldsymbol{\beta}=\mathbf{0}$. If $\alpha+\beta=0$, then (50) may be written as

$$
\left(1+\frac{\alpha \beta}{d^{2}}\right)\left[\frac{\sin (n \theta) \cos (\theta)}{\sin (\theta)}\right]+\left(1-\frac{\alpha \beta}{d^{2}}\right) \cos (n \theta)=0 .
$$

The cases $\alpha \beta=0$ and $\alpha \beta= \pm d^{2}$ have already been considered, so here we may assume that neither factor in parentheses is zero and these two factors are not equal. 

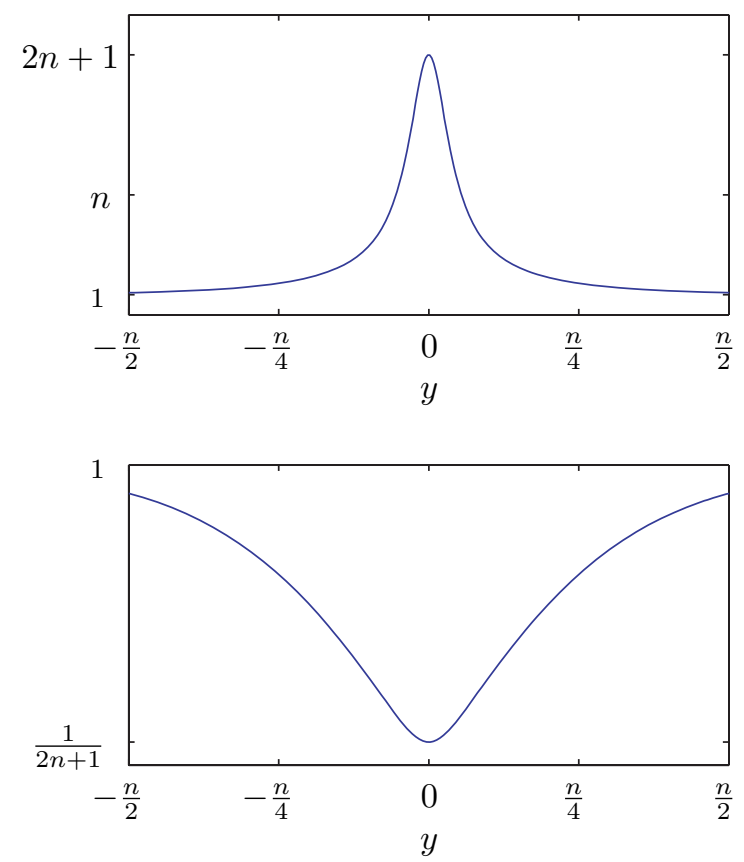

FIG. 5. Top: The function $y \mapsto \tanh ((2 n+1) y / 2) / \tanh (y / 2)$. Bottom: The function $y \mapsto$ $\operatorname{coth}((2 n+1) y / 2) / \operatorname{coth}(y / 2)$.

If $\cos (n \theta)=0$, then $\theta_{m}=\frac{(2 m-1) \pi}{2 n}, 1 \leq m \leq n$, are all of the possible values of $\theta$ in $[0, \pi]$. The factor $\sin (n \theta)$ clearly cannot be zero for these $\theta_{m}$; hence, for the equation to hold, $\cos \theta_{m}$ must be zero, that is, $\theta_{m}=\pi / 2$, which means that $n$ must be odd and $m=(n+1) / 2$. Thus the condition on $\theta$ may be written

$$
\frac{\tan (n \theta)}{\tan (\theta)}=\frac{\alpha \beta-d^{2}}{\alpha \beta+d^{2}}, \quad \theta \neq \pi / 2,
$$

where $\theta=\pi / 2$ is an additional solution in the case $n$ is odd. (Thus one eigenvalue of $A$ is always $b$ when $n$ is odd and $\alpha+\beta=0$.) Again, let $F(\theta)$ and $p(d, \alpha, \beta)$ be the left and right sides of (60), respectively, and suppose that the value of $p$ is real. Figure 6 displays a graph of $F$ on $[0, \pi]$ for both an even and an odd $n$ case. $F$ is even around $\pi / 2$ and is equal to $n$ at both $\theta=0$ and $\theta=\pi$. The subintervals of interest are the $I_{k}$ given by (47). On $I_{0}$ the function $F$ increases monotonically from $n$ to $+\infty$, and on intervals $I_{k}, 1 \leq k \leq n / 2-1$, it increases monotonically from $-\infty$ to $+\infty$. On the center interval(s) ( $I_{n / 2}$ if $n$ is even, $I_{(n-1) / 2} \cup I_{(n+1) / 2}$ if $n$ is odd $), F$ increases from $-\infty$ to a maximum value of zero at $\pi / 2$ and then decreases back to $-\infty$. The behavior on the other intervals is dictated by the fact that $F$ is even around $\pi / 2$. Thus if $p \geq n$, there is one real solution of (60) on each of the intervals $I_{k}, 0 \leq k \leq n / 2-1$, and $n / 2+1 \leq k \leq n$. If $p$ is smaller than zero, there is one real solution of (60) on each of the intervals $I_{k}, 1 \leq k \leq n / 2-1$, and $n / 2+1 \leq k \leq n-1$ and two in the center interval(s) ( $I_{n / 2}$ if $n$ is even, $I_{(n-1) / 2} \cup I_{(n+1) / 2}$ if $n$ is odd). This accounts for all $n$ solutions for $\theta$ (provided we add in the additional solution $\theta=\pi / 2$ if $n$ is odd). As $|p|$ gets large $\left(\alpha \beta \rightarrow-d^{2}\right)$, these solutions approach $\theta_{m}=(2 m-1) \pi /(2 n)$, as expected.

Now suppose that $0 \leq p<n$. First, if $1<p<n$, there is one real solution of (60) on each of the intervals $I_{k}, 1 \leq k<n / 2-1$, and $n / 2+1 \leq k \leq n-1$. By adding in 

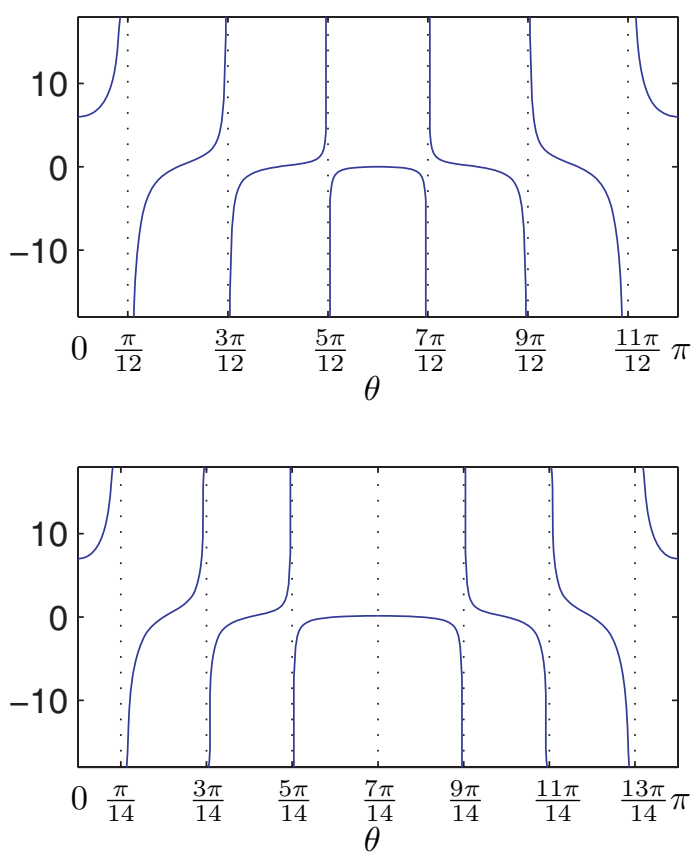

FIG. 6. The function $\theta \mapsto \tan (n \theta) / \tan (\theta), \theta \in[0, \pi]$, for an even case, $n=6$ (top), and an odd case, $n=7$ (bottom). The intervals $I_{k}, 0 \leq k \leq n$, are demarcated by the dotted vertical lines.

the solution $\theta=\pi / 2$ if $n$ is odd, this accounts for $n-2$ real solutions for $\theta$. However, a pair of complex solutions $\theta=i y$ and $\theta=\pi+i y$ exist, since both of these values for $\theta$, when substituted into $(60)$, yield

$$
\frac{\tanh (n y)}{\tanh (y)}=p,
$$

which has a unique solution $y^{*}$, with $y^{*}>0$, when $1<p<n$. (The plot of $\tanh (n y) / \tanh (y)$ is similar to that depicted in the top panel of Figure 5 but with maximum value $n$ rather than $2 n+1$.) It is impossible to have $d \neq 0$ and $p=1$, and, since we have dealt with $\alpha \beta=d^{2}$ previously, we have assumed that $p \neq 0$; however, we have not yet dealt with the case $0<p<1$. As $p$ increases through zero, two real roots annihilate each other at $\pi / 2$, and we therefore substitute $\theta=\pi / 2+i y$ into (60) yielding

$$
\frac{\tanh (n y)}{\operatorname{coth}(y)}=p .
$$

The function on the left side of (62) is plotted in Figure 7, where we note that there are two solutions $\pm y^{*}$ for each value of $p \in(0,1)$. These two solutions for $y$ correspond to two distinct values $\theta$ in $R$ and a pair of eigenvalues $\lambda$ given by

$$
\lambda=b \pm i 2 d \sinh \left(y^{*}\right) .
$$

If $b$ and $d$ are real, these are a complex conjugate pair.

3.2.4. $(\boldsymbol{\alpha}+\boldsymbol{\beta}) / \boldsymbol{d}$ and $\boldsymbol{\alpha} \boldsymbol{\beta} / \boldsymbol{d}^{2}$ are real. If the matrix parameters $d, \alpha$, and $\beta$ are such that the quantities $(\alpha+\beta) / d$ and $\alpha \beta / d^{2}$ are real, then we can conclude 


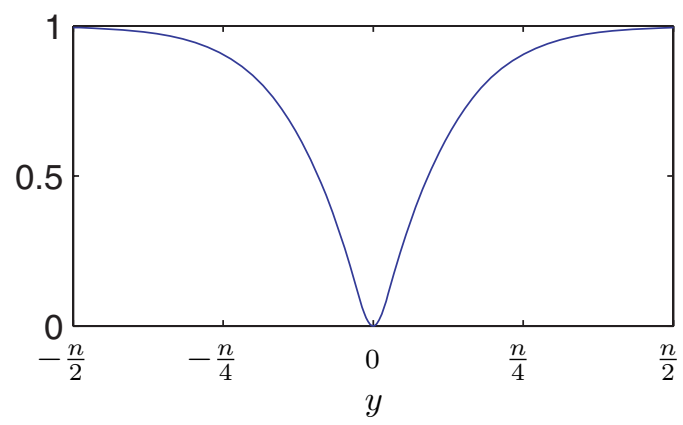

FIG. 7. The function $y \mapsto \tanh (n y) / \operatorname{coth}(y)$.

that there are at least $n-2$ real solutions to (20) and provide sufficient conditions under which $n$ real solutions exist. We shall assume that $\alpha \beta \neq d^{2}$ since that case was previously discussed.

The quantities $(\alpha+\beta) / d$ and $\alpha \beta / d^{2}$ are both real if and only if either both $\alpha / d$ and $\beta / d$ are real or they are complex conjugates, which we may express as one of the following two options:

1. $\alpha=s d, \beta=t d, s, t \in \mathbb{R}$,

2. $\alpha=(s+i t) d, \beta=(s-i t) d, s, t \in \mathbb{R}$.

Thus the manifold of points that we are considering is four-dimensional. Note that we have not restricted ourselves to the situation where the matrix is real, although that case is included as part of option 1 above.

Denote the left side of (20) (or equivalently the left side of $(50)$ ) as $T(\theta)$, so that the necessary and sufficient condition for $\theta$ is $T(\theta)=0$. From (20) and (16) we have

$$
\begin{aligned}
T(0) & =(n+1)+\frac{\alpha+\beta}{d} n+\frac{\alpha \beta}{d^{2}}(n-1) \\
& =\left(1+\frac{\alpha+\beta}{d}+\frac{\alpha \beta}{d^{2}}\right) n+\left(1-\frac{\alpha \beta}{d^{2}}\right)
\end{aligned}
$$

and

$$
\begin{aligned}
T(\pi) & =(1-)^{n}(n+1)+(-1)^{n-1} \frac{\alpha+\beta}{d} n+(-1)^{n-2} \frac{\alpha \beta}{d^{2}}(n-1) \\
& =(-1)^{n}\left[\left(1-\frac{\alpha+\beta}{d}+\frac{\alpha \beta}{d^{2}}\right) n+\left(1-\frac{\alpha \beta}{d^{2}}\right)\right] .
\end{aligned}
$$

Evaluating $T$ at $\theta=m \pi / n, 1 \leq m<n$, via (50) immediately gives

$$
T\left(\frac{m \pi}{n}\right)=(-1)^{m}\left(1-\frac{\alpha \beta}{d^{2}}\right) .
$$

Since we are assuming that $\alpha \beta \neq d^{2}$, the continuous function $T$ alternates sign at the points $m \pi / n, 1 \leq m<n$. This immediately implies that there are $n-2$ real roots of $T$, one in each of the intervals $K_{2}, K_{3}, \ldots, K_{n-1}$. The interval $K_{1}$ will contain an additional root provided $T(0)$ is zero or has opposite sign from $T(\pi / n)$, that is, $T(0) / T(\pi / n) \leq 0$. This can be expressed as

$$
\frac{1+\frac{\alpha+\beta}{d}+\frac{\alpha \beta}{d^{2}}}{1-\frac{\alpha \beta}{d^{2}}} \geq-\frac{1}{n} .
$$

Copyright $@$ by SIAM. Unauthorized reproduction of this article is prohibited. 
Analogously, the interval $K_{n}$ will contain an additional root provided

$$
\frac{1-\frac{\alpha+\beta}{d}+\frac{\alpha \beta}{d^{2}}}{1-\frac{\alpha \beta}{d^{2}}} \geq-\frac{1}{n}
$$

The inequalities (63) and (64) are sufficient conditions for a root to exist in $K_{1}$ or $K_{n}$, respectively. However, it is possible that two roots exist in one of these intervals if both inequalities fail to hold.

Substituting $\theta=i y$ or $\theta=\pi+i y, y \in \mathbb{R}$, into (50) yields the equivalent condition

$$
\frac{\tanh (n y)}{\sinh (y)}\left[\frac{1+\frac{\alpha \beta}{d^{2}}}{1-\frac{\alpha \beta}{d^{2}}} \cosh (y) \pm \frac{\frac{\alpha+\beta}{d}}{1-\frac{\alpha \beta}{d^{2}}}\right]=-1,
$$

where the $+\operatorname{sign}$ is for $\theta=i y$ and the $-\operatorname{sign}$ for $\theta=\pi+i y$. The function $y \rightarrow$ $\tanh (n y)(X \cosh (y)+Z) / \sinh (y)$ has limiting values of $n(X+Z)$ and $X$ as $y$ tends to 0 and $\infty$, respectively. However, it is not necessarily monotonic on $y>0$. Nonetheless, since it is continuous, we can conclude the following. If -1 lies between the values

$$
n\left(\frac{1+\frac{\alpha+\beta}{d}+\frac{\alpha \beta}{d^{2}}}{1-\frac{\alpha \beta}{d^{2}}}\right) \quad \text { and } \quad \frac{1+\frac{\alpha \beta}{d^{2}}}{1-\frac{\alpha \beta}{d^{2}}},
$$

there will be at least one solution of $(65)$ in the form $\theta=i y, y \in \mathbb{R}$. If -1 lies between the values

$$
n\left(\frac{1-\frac{\alpha+\beta}{d}+\frac{\alpha \beta}{d^{2}}}{1-\frac{\alpha \beta}{d^{2}}}\right) \quad \text { and } \quad \frac{1+\frac{\alpha \beta}{d^{2}}}{1-\frac{\alpha \beta}{d^{2}}},
$$

there will be at least one solution of (65) in the form $\theta=\pi+i y, y \in \mathbb{R}$. (Note that either of the two values in each of the above pairs may be the smaller one, depending on $d, \alpha$, and $\beta$.)

4. Conclusion. The eigenvalues of the tridiagonal matrix $A$ given by (1) are of the form $\lambda_{m}=b+2 d \cos \left(\theta_{m}\right)$, where $\theta_{m}$ are the solutions to the nonlinear equation (20) in the region $R$ defined by (21). The corresponding eigenvectors are given by (24).

The space of matrix parameters $d, \alpha$, and $\beta$ is $\mathbb{C}^{3}$, which can be identified with $\mathbb{R}^{6}$. Restriction to a number of two-dimensional manifolds in this space permits explicit solutions of (20), and these cases were itemized. In addition, the four-dimensional manifold of points $\alpha \beta=d^{2}$ also yields explicit solutions, and these were given. Information about the distribution of eigenvalues was also described for several other three- and four-dimensional manifolds. Many of these were cases where (20) could be separated into the form $F(\theta)=p(d, \alpha, \beta)$, where $F$ is a certain ratio of trigonometric functions of multiples of $\theta$ and $p$ is real-valued. In most of these cases, this equation has either $n$ real roots or $n-1$ real roots and one complex root, but the corresponding eigenvalues are all real (assuming that $b$ and $d$ are also real). In one case, $\alpha+\beta=0$, a single complex conjugate pair of eigenvalues is possible if $0<\left(\alpha \beta-d^{2}\right) /\left(\alpha \beta+d^{2}\right)<1$. The four-dimensional manifold of points specified by the requirement that both the quantities $(\alpha+\beta) / d$ and $\alpha \beta / d^{2}$ be real was shown to yield at least $n-2$ real eigenvalues for $A$. Sufficient conditions for when the remaining two eigenvalues are also real were provided. This case includes the case where the matrix $A$ is real to begin with.

Efficient numerical algorithms exist, based on the QR factorization, for the computation of the eigenvalues of general tridiagonal matrices [2]. The nonlinear equation (20) we have presented here would generally need to be solved with a root-finding 
algorithm to find the $\theta_{m}$ in the region $R$, which may or may not be as efficient as the QR-based algorithms. However, the various special cases that we have enumerated provide additional information on the distribution of the solutions $\theta_{m}$, specifying various subintervals of $[0, \pi]$ wherein exactly one solution must lie. Within these subintervals, more refined estimates of the locations of solutions are often easily obtained by plotting the appropriate function $F$ described herein and noting where it crosses the value of $p$.

Tridiagonal matrices frequently occur in applications where it is desirable to know their eigenvalues. As just one example, consider a simple Markov process with $n$ states arranged in a chain formation where $x_{i}(t)$ is the probability of being in state $i$ at time $t, i=1, \ldots, n$. Let $a$ be the rate at which a particle moves to the right (from state $i$ to state $i+1)$ and $c$ the rate at which a particle moves to the left through the states:

$$
x_{1} \underset{c}{\stackrel{a}{\rightleftarrows}} x_{1} \underset{c}{\stackrel{a}{\rightleftarrows}} x_{2} \cdots x_{n-1} \underset{c}{\stackrel{a}{\rightleftarrows}} x_{n} .
$$

Such systems are commonly employed as models or parts of models for ion channel gating [1]. The governing system is

$$
x^{\prime}=A x,
$$

where $A$ is given by (1) with $b=-a-c, d=\sqrt{a c}, \alpha=-c$, and $\beta=-a$. From (40) we immediately conclude that the eigenvalues are

$$
\lambda_{m}=-(a+c)+2 \sqrt{a c} \cos \left(\frac{m \pi}{n}\right), \quad 1 \leq m<n, \quad \lambda_{n}=0 .
$$

From (7), (45), and (46), the corresponding eigenvectors are

$$
v_{j}^{(m)}=\left(\frac{a}{c}\right)^{(j-1) / 2}\left[\sin \left(\frac{j m \pi}{n}\right)-\sqrt{\frac{c}{a}} \sin \left(\frac{(j-1) m \pi}{n}\right)\right], \quad 1 \leq j \leq n, 1 \leq m<n,
$$

and

$$
v_{j}^{(n)}=\left(\frac{a}{c}\right)^{(j-1) / 2}\left[\sin \left(j \theta_{n}\right)-\sqrt{\frac{c}{a}} \sin \left((j-1) \theta_{n}\right)\right], \quad 1 \leq j \leq n,
$$

where $\theta_{n}=\arccos ((a+c) / 2 \sqrt{a c})$.

\section{REFERENCES}

[1] F. Bezanilla, The voltage sensor in voltage-dependent ion channels, Physiol. Rev., 80 (2000), pp. 555-592.

[2] R. L. Burden and J. D. Faires, Numerical Analysis, 7th ed., Brooks/Cole, Forest Grove, CA, 2001.

[3] S. S. Cheng, Partial Difference Equations, CRC Press, New York, 2003.

[4] C. M. DA FonseCA, The characteristic polynomial of some perturbed tridiagonal $k$-Toeplitz matrices, Appl. Math. Sci., 1 (2007), pp. 59-67.

[5] C. M. DA Fonseca, On the eigenvalues of some tridiagonal matrices, J. Comput. Appl. Math., 200 (2007), pp. 283-286.

[6] S. KouachI, Eigenvalues and eigenvectors of tridiagonal matrices, Electron. J. Linear Algebra, 15 (2006), pp. 115-133.

[7] W. Ledermann and G.E.H. Reuter, Spectral theory for the differential equations of simple birth and death processes, Philos. Trans. R. Soc. Lond. Ser. A, 246 (1954), pp. 321-369.

[8] C. D. Meyer, Matrix Analysis and Applied Linear Algebra, SIAM, Philadelphia, PA, 2000.

[9] W.-C. YueH, Eigenvalues of several tridiagonal matrices, Appl. Math. E-Notes, 5 (2005), pp. $66-74$. 